\title{
Bioanalysis
}

\section{Tiered approach into practice: scientific validation for chromatography- based assays in early development - a recommendation from the European Bioanalysis Forum}

The principles of tiered approach have been part of the bioanalytical toolbox for some years. Nevertheless, an in spite of many valuable discussions in industry, they remain difficult to apply in a harmonized way for a broad array of studies in early drug development where these alternative approaches to regulated validation would make sense. The European Bioanalysis Forum has identified the need to proposes some practical workflows for five categories of studies for chromatography based assays where scientific validation will allow additional freedom while safeguarding scientific rigor and robust documentation: quantification of metabolites in plasma in relation to ICH M3(R2), urine analysis, tissue homogenate analysis, and preclinical and clinical studies in early stages of drug development. The recommendation would introduce a common language and harmonized best practice for these study categories and can help to refocus towards optimized scientific and resource investments for bioanalysis in early drug development.

Keywords: early clinical development $\bullet$ early preclinical development $\bullet$ European Bioanalysis Forum $\bullet$ metabolites $\bullet$ scientific validation $\bullet$ tiered approach $\bullet$ tissue analysis $\bullet$ urine analysis

The principles of tiered approach need little introduction in the bioanalytical community. Since it became part of the bioanalytical language during the Crystal City III discussions [1], many papers have been published by international consortia $[2,3]$ or individual researchers [4-6] describing its benefit as a valid alternative for applying the full validation workflows as described in different regulatory guidance documents [7-9]. Two common themes in many of the publications are that: the increasing pressure on the industry to deliver drugs faster and more cost effective to the patients: and the development of new applications in support of a diversifying biological and chemical portfolio in drug development require a different view on bioanalytical method establishment and application of those methods in a broad array of assay formats. Advances in technologies across all aspects of bioanalysis, for example, micro sampling or hybrid assays for analysis of peptides and proteins are nice examples of how alternative validation approaches compared with the workflows in regulatory guidance will enable industry to embrace technological and scientific progress more rapidly. The European Bioanalysis Forum (EBF) has been a strong proponent in helping to create a sustainable environment to support a mindset shift in industry. In this, EBF has observed the bioanalytical community strictly adhere to the aforementioned Guidelines as 'normal practice' for bioanalysis, irrespective of the purpose for which they were intended or designed. As such, many feel constrained by the Guidelines and unable to apply the appropriate scientific freedom and resources needed for efficient bioanalysis appropriate for many activities required during early drug development. Previously at the AAPS Annual meeting in Los Angeles (November 2009), and building on the CC-III discussions, the EBF stimulated the industry to intensify the use of the principles of tiered approach by proposing three levels of method
Philip Timmerman $*, 1$, Stephen White ${ }^{2}$, Stuart McDougall ${ }^{3}$, Morten A Kall ${ }^{4}$, John Smeraglia ${ }^{5}$, Marianne Scheel Fjording ${ }^{6} \&$ Magnus Knutsson ${ }^{7}$

'Janssen R\&D, Turnhoutseweg, 30, B-2340 Beerse, Belgium

${ }^{2}$ GlaxoSmithKline, Ware, UK ${ }^{3}$ Covance, Alnwick, UK ${ }^{4} \mathrm{H}$ Lundbeck A/S, Copenhagen, Denmark ${ }^{5} U C B$ BioPharma, Braine-l'Alleud, Belgium

${ }^{6}$ Novo Nordisk A/S, Måløv, Denmark ${ }^{7}$ Ferring Pharmaceuticals A/S, Copenhagen, Denmark

*Author for correspondence:

Tel.: +32 14603581 ptimmerm@its.jnj.com 
validation. At that time the discussion was focused on the bioanalytical workload related to metabolite quantification as part of ICH M3 (R2) [10], work which was published in 2010 [2]. The discussion was broadened as part of an EBF Workshop in 2011 discussing the challenges in regulated bioanalysis in relation to the integration of innovation and new technologies in regulated bioanalysis [11,12].

Two important catalysts followed to continue the discussion and add tiered approach into the toolbox of the bioanalytical scientist; a paragraph in the recent (draft) Regulatory Guidance documents from the US FDA [13] and the MHLW [3]. Although these provided appropriate rationale to apply alternative workflows, neither this nor aforementioned publications by EBF [2] and GBC [3] gave the bioanalytical community sufficient practical details on how to apply tiered approach in practice and in a harmonized way across industry. In addition, the bioanalytical community has become more risk averse over the last decade, stimulating laboratories to apply regulatory guidance workflows for studies where they do not allow an optimal scientific focus. This has proved to be a limiting factor to apply tiered approach principles in studies where they can make a difference with respect to science and resources spent.

A special issue of Bioanalysis on tiered approach [14] highlighted many areas where tiered approach can be a valid alternative for regulated bioanalytical standards. Advancing the ideas expressed in this special issue, and building on a commentary paper [15], the EBF sponsored a Focus Workshop in 2014 [16]. At the workshop, an intense discussion was held to come to a draft proposal on practical workflows and acceptance criteria for five important areas in early drug development, described in the next paragraphs, to apply tiered approach in favor of current practice where the industry often uses full assay validation. Results of the workshop and the continued discussion of the EBF team were presented in the 7th EBF Open Symposium [17] and at a joint EBF/Delaware Valley Drug Metabolism discussion group (DV-DMDG) meeting [18] .

\section{Scientific validation \& scope of the EBF recommendation}

Applying more liberal analytical workflows in a discovery phase is common practice in industry. These assays are often based upon the basic principles of bioanalysis (i.e., some form of calibration curves or QC and stability assessment, restricted sampling conditions to manage potential instability among others) and tuned to allow rapid turnaround while safeguarding appropriate quality for decision-making. The workflows used for these discovery-type studies, in the GBC paper referred to as screening or research workflows, are not in scope in this manuscript.

In this manuscript, the EBF wishes to recommend alternative scientific method establishment criteria which in previous publications are part of the area of qualified assays. In practice, however, we continue to see hesitation for full adoption by our industry of these principles because for many 'qualified' evokes 'poor/less quality' and 'cutting corners.' Hence, we see value or more accurately describe these workflows into 'scientific validation' to express their goal to deliver fully scientifically valid concentration data for robust decision-making.

In line with the discussions at the 2014 Focus Workshop, our proposals are on five types of matrices/study types where the industry disagrees or hesitates whether regulatory quality level is required, or there is doubt whether the Regulatory Guidance workflows are the best guarantee to generate accurate concentrations. As a consequence, the risk remains that we spend too much resource in supporting sample analysis for these five areas of matrices/study types. The five areas for which EBF is proposing scientific validation criteria are:

- Quantification of metabolites in plasma in relation to ICH M3(R2): as part of the experiments to document to what extent the exposure of metabolites in man is covered in the animal species, many laboratories are setting up validated assays using the regulated bioanalytical templates for metabolites in early stages of development even before the relevance of understanding metabolite exposure in human plasma is required. As a consequence, many resources are spent on assay validation, synthesis of (often stable labeled) IS for metabolites with no pharmacological or safety related contribution once the ICH M3(R2) criteria on relative exposure to man is documented [24]. Therefore, optimizing all metabolite quantification in early development using scientific validation criteria will facilitate a more harmonized approach to metabolite quantification with optimized use of resources. In essence, this approach has already been endorsed as part of the Crystal City III discussions, albeit assay criteria were never discussed in detail;

- Urine analysis (in all stages of development): urine analysis is typically performed as a scientific parameter to document the renal excretion of the dosed drug or its metabolites. Although this study is mostly executed during early stages of development, the EBF recommends using scientific validation workflows for urine analysis in all stages of development, because the bioanalytical challenges of urine analy- 
sis are different from plasma analysis. Depending on the metabolism, excretion pathway and, from a bioanalytical perspective, the $\log \mathrm{D}$ of the compound, the major challenge of urine analysis may be focused on the sampling and storage conditions of urine;

- Tissue (homogenate) analysis (in all stages of development): similar to urine analysis, tissue samples are usually analyzed as a scientific parameter to document and often focus on the (relative) distribution of a drug or its metabolites in different tissues or to calculate relative exposure ratios with plasma. It should be noted that the bioanalytical laboratory in essence reports tissue homogenate concentration. In addition to the proposed workflow, more indepth discussion on tissue (homogenate) analysis are summarized in [19] and [20];

- Clinical studies in early stages of drug development: for pivotal studies that require regulatory action for approval or labeling, there is no challenge to use validation criteria per Guidance. But, in the current paradigm of applying the regulatory workflow for full assay validation of clinical studies in early stages of drug development, for example, the First into Human studies, the bioanalytical scientist is validating the drug assay without knowing some essential pieces of the puzzle needed to set up the right assay. In absence of historic data in man, the only basis for deciding on the details of the assay (e.g., calibration range and metabolites) are animal data and/or modeling and simulation data at most. Taking into consideration that LC-MS/MS assays typically have a dynamic range of three decades, having a fixed validation range as part of the full validation will require multiple partial validations in between dose escalation or reassays of diluted samples. Hence, and in view of the fact that the proposed criteria for scientific validation are designed to deliver quality data to make valid decisions, it makes more sense to delay the full regulatory validation until more knowledge is gathered on the dose range, PK and metabolic behavior of the compound in man. In line with the current workflows in industry in support of ICH M3 (R2), a more appropriate moment to set up a regulatory validated assay would be after the multiple ascending dose or around proof of concept. At that time, most of the pieces of the puzzle should be available to set up an assay which can serve the expected dose range considering the metabolism or anticipated co-medication. At these milestones, the bioanalytical laboratory will be in a better place to fully validate an assay which can continue to be used in the further development stages of the drug;
- Early development preclinical studies: for many early development non-GLP studies, laboratories are already considering tiered approach. As part of the discussion within EBF and the surveys we performed, a broader array of early development studies were identified to fall into the category qualifying for scientific validation, including the early toleration studies, Dose Range Finders and even the first (28d) GLP studies. The latter may come as a surprise for many, but when looking at the GLP regulations, using scientific validation would be in line with GLP. From a GLP perspective, the bioanalytical scientist is required to support bioanalysis for TK as part of GLP studies with assay validation criteria that are predefined in a protocol or SOP, and in continuation, he/she needs to adhere to this protocol/ SOP and document all actions in accordance to GLP regulations. However, from a scientific perspective, the assay validation criteria described in the bioanalytical Guidance documents may not fully serve the requirements for toxicokinetics in preclinical studies. By adding early GLP studies to the list of studies qualifying for scientific validation, the EBF wants to knock on the door for a discussion on how we can optimize the support for these first GLP studies in view that we may be overinterpreting application of the current bioanalytical Guidances for use in GLP studies and that, as a consequence, the bioanalytical laboratory is often on the critical path to deliver these methods in time for TK support.

In line with [15], the first three categories are referred to as assay-appropriate scientific validation (i.e., irrespective of the development stage in which the study is performed, the proposed validation criteria support valid and documented decision-making from the reported concentrations). Categories 4 and 5 are referred to as stage-appropriate scientific validation (i.e., depending on the development stage in which the study is performed, the proposed validation criteria may vary).

We cannot emphasize strongly enough that by introducing the terminology of scientific validation for the five categories mentioned, the EBF does not intend to undermine the scientific foundations of Regulatory Guidance workflows applied for bioanalytical support in later stages of development or for all pivotal studies that require regulatory action for approval or labeling, such as BE or PK studies. In fact, these Guidance were built on the solid scientific experience for later stage clinical studies for which they were initially intended by many experts from industry and regulators. As highlighted earlier, they may not necessarily fit the current drug development needs in areas outside late stage clinical. 
Finally, by proposing criteria for scientific validation, the EBF wants to promote the best bioanalytical practices which have been the foundation of bioanalysis for more than 25 years. Continuing on the work started in [2], we identified some 30 parameters to consider for pre- or in-study validation in relation to scientific validation. In addition, each of the five categories may require some additional and specific scientific experiments, which will be discussed in later paragraphs.

When reviewing the criteria for scientific validation against current guidance one might question if there is real added value to propose a variation on an established theme. Most parameters from the Guidance are maintained as required scientific data in scientific validation, so why the proposed change? At closer evaluation, a few important differences surface; acceptance criteria are relaxed, size and number of replicates required to come to a validated status are lowered. Refinements are proposed to help the bioanalytical scientist focus on the intended purpose with respect to quality and reconstructability of the studies related to internal decision-making in projects in early stages of development. Maybe the most important waiver in scientific validation is the possibility to limit the prestudy validation to a minimum and include validation criteria as part of in-study validation. Indeed, the possibility to consider in-study validation (using a priori defined acceptance criteria), entirely or for certain parameters (e.g., stability and dilution integrity) should allow prestudy validation experiment to be limited to one single run. To that end, combining stability experiments and simplify and/or widening the P\&A acceptance criteria can save resources without compromising valid decision-making from the data generated.

During our discussion in the EBF community and at meetings, we listened to the reflections that, at later stages, a bioanalytical laboratory may need to set up a full assay validation after all, doubting the relevance of saving a few days of time in early phases of development. So, why create a second set of standards? Indeed, the proposed time saving can be challenged from a single compound/project perspective, where they become irrelevant in view of the total project costs. But when looking at attrition rates in pharma $\mathrm{R} \& \mathrm{D}$, less than $5 \%$ of compounds that reach clinical Phase I or only $10 \%$ of molecules that enter Phase II will finally reach the market [21], any time/cost saving in early phases of development should be multiplied at least by a factor of 20 as a counter argument to the one challenging tiered approach as an invitation for repetition of work in later stages. In addition and as discussed at the recent DV-DMVG meeting [18] most scientific bioanalytical parameters, like assay range, robustness, stability, analytical parameters, co-medications, metabolism or sampling logistics can be documented at the end of Phase II. As a consequence, consolidating all scientific and regulatory validation experience available at that point into a fresh assay, fine-tuned for the continuation of clinical program at the start of Phase III makes sense. Hence, some repetition of assay validation efforts at a later stage to include final requirement for a drug coming closer to success may be a good investment in preparation of transparency in filing.

In Tables 1 \& 2, the proposed criteria for prestudy and in-study validation are summarized for assay appropriate scientific validation. In Tables $3 \& 4$, the proposed criteria for pre- and in-study validation are summarized for the analysis as part of stage appropriate scientific validation.

\section{Discussion}

\section{Reference standards}

At the time of the execution of studies in scope for scientific validation, in view of the early stage of development of the studies in scope, full coverage of the stability of the reference material may not be available. The information on the CoA may be limited to a retest date rather than an expiry date. EBF would consider this to be acceptable for scientific validation. In absence of a CoA with an expiry date, we would recommend to use the same batch that was used for dosing also for preparing calibration standards and QCs to compensate for potential purity differences of different batches of drug product in early stages of development wherever possible.

For metabolites, the situation may be similar and the same level of documentation may not be available as for the dosed drug. Most metabolite analysis in scope of ICH M3 (R2) are performed in early stages of development. The focus is on documenting relative exposure of metabolites, comparing exposure in preclinical species to man, rather than requiring absolute concentration values (i.e., expressed in $\mathrm{ng} / \mathrm{ml}$ or similar). Therefore, the EBF recommends using the same reference material for calibration in animals and human for this comparison. When metabolites need to be quantified to understand the anticipated significant contribution to the pharmacological effect, we would expect a reference compound to be available and recommend the use thereof for calibration purposes. For this reference compound, at least data on identity and the purity should be available. It is likely that stability (expiry date) is not available, and a retest date should be sufficient.

\section{Calibration curves \& QCs}

The acceptance criteria for calibration curves, calibrators and related parameters for pre- or in-study valida- 
tion can be found in Tables 1-4. In essence, for scientific validation we propose slightly more relaxed acceptance criteria compared with regulatory validation. For the studies in scope and the decision made using the data from these studies, we consider that the validity of the decision would not be compromised by this.

We recommend including a minimum of three levels of QC in duplicate per analytical run and with nominal concentrations of the L/M/H QC inspired by the Guidance. However, because of the increased freedom with respect to the final calibration range of each batch, there may be instances where the lowest $\mathrm{QC}$ level is at the same nominal concentration as the lowest calibration sample passing with acceptable accuracy and precision (i.e., 25\% in case of '4-6-20' criteria). In these cases, an analytical batch would pass if the QC sample at this nominal concentration would have similar accuracy and precision.

There has been a lot of discussion and anxiety on the real impact of these alternative acceptance criteria and whether or not this will impact the quality of the concentration data generated. This discussion may be more philosophical than scientific. The impact of trying to develop a method that can reach ' $4-6-15$ ' criteria instead of, for example, '4-6-20' is difficult to quantify, but it is easy to imagine that developing a method that will consistently meet ' $4-6-15$ ' may include taking some additional measures (e.g., additional robustness testing and repetition of prevalidation experiments) to limit or prevent failed runs during validation or production. These additional measures may not be needed or at least decreased when developing a method with slightly relaxed criteria. However, it is important to understand that a method validated towards ' $4-6-15$ ' will yield data that will at least pass these acceptance criteria when used in production. In most instance, the actual accuracy and precision will be better that "4-615'. In the same way, this will be the case for assays that are developed to meet at least '4-6-20'. Experience shows that study samples analyzed and passing these ' $4-6-20$ ' criteria will mostly pass the ' $4-6-15$ ' mark as well and that only a small percentage will land between 15 and $20 \%$. Therefore, we should not look at the quality of a study in respect to the assay validation criteria, but instead focus on the performance of the method during production. Hence, the real advantage of using a method with more relaxed acceptance criteria lies in the fact that less time may need to be spent in setting up the assay. The additional gain, albeit unlikely to occur often, includes not requiring reanalysis when the method performs in between ' $4-6-15$ ' and '4-6-20'. Using relaxed criteria should be agreed on beforehand and it should not jeopardize the scientific value of the decision made with the data.

\section{Extrapolation of below lowest calibration point or over the curve samples}

In comparison with regulatory validation, EBF suggests that limited extrapolations of concentration for study samples outside of the nominal calibration range in line with accuracy and precision criteria proposed for the different assay types is acceptable. As an example, an assay with a calibration range from 1.00 to $1000 \mathrm{ng} / \mathrm{ml}$ and using '4-6-20(25)' criteria, would accept a back-calculated values for CAL and QC from 0.75 to $1.25 \mathrm{ng} / \mathrm{ml} \mathrm{(at} 1 \mathrm{ng} / \mathrm{ml}$ ) and from 800 to $1200 \mathrm{ng} / \mathrm{ml}$ (at $1000 \mathrm{ng} / \mathrm{ml}$ ). Hence, extrapolation of the calibration range for study samples, in accordance with the predefined accuracy criteria of the calibration line, and considering acceptable $S / N$, would be scientifically justifiable for a linear model.

\section{Stability}

Just as for regulatory validation, stability is a key quality parameter for any bioanalytical assay. In essence, EBF recommends investigating and documenting all relevant stability data required to make valid decisions from the data. However, in contrast to the regulatory validation workflows, some of the stability experiments may be combined and/or be integrated as part of in-study validation, presenting a considerable saving of time and effort. Data supporting the stability of the samples or stock solutions used should be available at the time of reporting of the data. There may be cases that stability data can be gathered from scientific evaluation of the analytical data not necessarily intended for this purpose. If this evaluation can be defended using scientific arguments, no additional separate experiments may be required to document single stability data.

\section{Extraction recovery}

EBF does not consider extraction recovery to be a required assay validation parameter as absolute recovery is not critical. Consistency/reproducibility of recovery will be evident from prestudy validation and/or within study assay performance.

\section{Carry over}

Although carry over assessment will likely be part of method development, EBF recommends to document carry over only for sample analysis. EBF does not provide nominal criteria to evaluate carry over, but would expect a scientific evaluation to ensure any undue carry over does not impact the decisions taken from the data. These criteria can be different depending on study type. In contrast to other acceptance criteria, carry over assessment would be allowed post analysis, but documentation should allow scientific scrutiny. 
Table 1. Proposed criteria for pre-study method validation for the analysis of metabolites (in relation to ICH M3 [R2]), urine and tissue analysis.

\begin{tabular}{|c|c|c|c|}
\hline \multirow[t]{2}{*}{ Parameter } & \multicolumn{3}{|c|}{ Assay appropriate scientific validation } \\
\hline & Metabolites in plasma (ICH-M3) & Urine & Tissue homogenates \\
\hline $\begin{array}{l}\text { CoA with at minimum proof } \\
\text { of identity/purity }\end{array}$ & $\mathrm{N}$ & Y or use dosed batch & Y or use dosed batch \\
\hline $\begin{array}{l}\text { Calibration curve: number of } \\
\text { calibration samples }\end{array}$ & $\begin{array}{l}\text { Minimum } 5 \text {, covering the ranges } \\
\text { of incurred samples }\end{array}$ & $\begin{array}{l}\text { Minimum } 5 \text {, covering the } \\
\text { ranges of incurred samples }\end{array}$ & $\begin{array}{l}\text { Minimum } 5 \text {, covering the } \\
\text { ranges of incurred samples - } \\
\text { surrogate matrix acceptable }\end{array}$ \\
\hline Acceptance criteria CAL & $\begin{array}{l}75 \% \text { and at least } 5 \text { points within } \\
20 \%(25 \% \text { at LLOQ })\end{array}$ & $\begin{array}{l}75 \% \text { and at least } 5 \text { points } \\
\text { within } 25 \%(30 \% \text { at LLOQ }\end{array}$ & $\begin{array}{l}75 \% \text { and at least } 5 \text { points } \\
\text { within } 25 \%(30 \% \text { at LLOQ }\end{array}$ \\
\hline Matrix QC identical as study & $\mathrm{Y}$ & Y (or matrix matching) & Y (or matrix matching) \\
\hline QC levels - replicates & 3 (low/mid/high) - min 3 reps & 3 (low/mid/high) - min 3 reps & 3 (low/mid/high) - min 3 reps \\
\hline $\begin{array}{l}\text { Acceptance criteria QC - } \\
\text { mean bias }\end{array}$ & $20 \%$ & $25 \%$ & $25 \%$ \\
\hline $\begin{array}{l}\text { Acceptance criteria QC (\%CV } \\
\text { per level) }\end{array}$ & $20 \%$ & $25 \%$ & $25 \%$ \\
\hline Inter assay variability & $\begin{array}{l}\text { Use scientific judgment based } \\
\text { upon P\&A of 1-run validation }\end{array}$ & $\begin{array}{l}\text { Use scientific judgment based } \\
\text { upon P\&A of 1-run validation }\end{array}$ & $\begin{array}{l}\text { Use scientific judgment based } \\
\text { upon P\&A of 1-run validation }\end{array}$ \\
\hline QC/CAL from separate stocks & $\mathrm{N}$ & Y (unless check equivalence) & Y (unless check equivalence) \\
\hline Selectivity & $\begin{array}{l}\text { Minimum one source of blank } \\
\text { matrix (as used for CALs/QCs) }\end{array}$ & $\begin{array}{l}\text { Minimum one source of blank } \\
\text { matrix (as used for CALs/QCs) }\end{array}$ & $\begin{array}{l}\text { Minimum one source of blank } \\
\text { matrix (as used for CALs/QCs) } \\
\text { - multiple sources depending } \\
\text { on practicality }\end{array}$ \\
\hline Extraction recovery & $\mathrm{N}$ & $\mathrm{N}$ & $\mathrm{N}$ \\
\hline Carryover & In study & In study & In study \\
\hline Matrix effect & $\begin{array}{l}\mathrm{N} \text {, assess within study runs via IS } \\
\text { response }\end{array}$ & $\begin{array}{l}\mathrm{N} \text {, assess within study runs via } \\
\text { IS response }\end{array}$ & $\begin{array}{l}N \text {, assess within study runs via } \\
\text { IS response }\end{array}$ \\
\hline Dilution integrity & In study & In study & In study \\
\hline LLOQ & $\begin{array}{l}\text { As defined by acceptable LLOQ } \\
\text { CAL standard }\end{array}$ & $\begin{array}{l}\text { As defined by acceptable } \\
\text { LLOQ CAL standard }\end{array}$ & $\begin{array}{l}\text { As defined by acceptable CAL } \\
\text { LLOQ standard }\end{array}$ \\
\hline $\begin{array}{l}\text { Comed selectivity (in support } \\
\text { of DDI studies) }\end{array}$ & $\mathrm{N}$ & $\mathrm{N}$ & $\mathrm{N}$ \\
\hline Over the counter stability & $\mathrm{N}$ & $\mathrm{N}$ & $\mathrm{N}$ \\
\hline $\begin{array}{l}\text { Fixed sose combination } \\
\text { stability }\end{array}$ & $\mathrm{N}$ & $\mathrm{N}$ & $\mathrm{N}$ \\
\hline $\begin{array}{l}\text { Processed sample stability/ } \\
\text { reproducibility }\end{array}$ & $\mathrm{N}$ & $\mathrm{N}$ & $\mathrm{N}$ \\
\hline Stock solution stability & $20 \%-$ minimal assessment & If available previously & If available previously \\
\hline Bench-top stability & $\mathrm{N}$ & $\mathrm{N}$ & $\mathrm{N}$ \\
\hline $\begin{array}{l}\text { Sample stability for duration } \\
\text { of storage }\end{array}$ & $Y(20 \%)$ & $\mathrm{N}$ & $\mathrm{N}$ \\
\hline F/T stability & $\mathrm{N}$, consider ISS & Y (1 cycle) & Y (1 cycle) \\
\hline Whole blood stability & $\begin{array}{l}\mathrm{N} \text {, unless for known problem } \\
\text { scaffolds, }[22]\end{array}$ & N/A & $\mathrm{N} / \mathrm{A}$ \\
\hline Sampling conditions & $\mathrm{N}$ & $\begin{array}{l}\text { Y, consider including } \\
\text { container and adsorption }\end{array}$ & $\begin{array}{l}\text { Y, specify conditions, consider } \\
\text { EBF paper }[20]\end{array}$ \\
\hline
\end{tabular}




\begin{tabular}{|c|c|c|c|}
\hline \multirow[t]{2}{*}{ Parameter } & \multicolumn{3}{|c|}{ Assay appropriate scientific validation } \\
\hline & Metabolites in plasma (ICH-M3) & Urine & Tissue homogenates \\
\hline Hemolytic & $\mathrm{N}$ & N/A & \\
\hline Hyperlipidemic & $\mathrm{N}$ & N/A & $\mathrm{N} / \mathrm{A}$ \\
\hline Validation plan/protocol & $\begin{array}{l}\text { At minimum SOP or short } \\
\text { protocol summarizing scientific } \\
\text { parameters to be tested }\end{array}$ & $\begin{array}{l}\text { At minimum SOP or short } \\
\text { protocol summarizing } \\
\text { scientific parameters to be } \\
\text { tested }\end{array}$ & $\begin{array}{l}\text { At minimum SOP or short } \\
\text { protocol summarizing } \\
\text { scientific parameters to be } \\
\text { tested }\end{array}$ \\
\hline Validation report & $\begin{array}{l}\text { At minimum a document } \\
\text { summarizing scientific } \\
\text { parameters tested }\end{array}$ & $\begin{array}{l}\text { At minimum a document } \\
\text { summarizing scientific } \\
\text { parameters tested }\end{array}$ & $\begin{array}{l}\text { At minimum a document } \\
\text { summarizing scientific } \\
\text { parameters tested }\end{array}$ \\
\hline Misc. & $\begin{array}{l}\text { Consider longer run time instead } \\
\text { of specificity experiment }\end{array}$ & $\begin{array}{l}\mathrm{n}=\text { during meth dev. Non- } \\
\text { specific binding/sampling } \\
\text { conditions and aliquoting }\end{array}$ & $\begin{array}{l}\text { Calibration matrix may be a } \\
\text { surrogate }\end{array}$ \\
\hline
\end{tabular}

\section{ISR}

In the context of scientific validation as part of this recommendation paper, EBF proposes that ISR is not required as part of sample analysis performed for studies belonging to assay appropriate scientific validation.

For stage appropriate scientific validation, EBF would recommend to perform ISR for the first time the method is used. However, we would recommend limiting the number of samples to 20 [24] and use acceptance criteria in line with current EMA Guidance.

\section{Matrix effects \& IS variability}

Although matrix effect is an important parameter for (LC-MS/MS) bioanalysis, the EBF would not consider testing matrix effects as described in the EMA Guidance to be required. The potential impact of matrix effects on the scientific validity of the study results can be assessed using the IS response variability using acceptance criteria based on a recent EBF recommendation paper [23] and defined a priori.

\section{Dilution integrity}

The EBF recommends to document dilution integrity as part of in-study validation.

\section{Chromatography}

In general, and considering limited knowledge of coeluting interfering peak and/or metabolites the EBF recommends considering using longer run times and higher separation power of the LC. Typically, we would recommend a minimal K-value of 3-5 to safeguard selectivity and prevent undue matrix effects or co-eluting interferences negatively affecting the data.

\section{Additional considerations for specific assay types}

In addition to the above general principles of scientific validation recommended for all assay types described in this manuscript, some special areas of focus are worthwhile highlighting.

\section{Metabolites \& nonpivotal early clinical}

Although hemolytic and hyperlipemic samples may impact the scientific validity of the reported concentrations, we consider the safety net as part of IS variability sufficient to waive the need for additional testing for hemolytic and hyperlipemic samples.

\section{Urine}

Taking into account the specific challenge of adsorption of (often basic and relative lipophilic) drugs to containers used during sampling and aliquoting of urine samples, we recommend to document nonspecific binding, adsorption or other effects with sufficient detail to be a key area of focus for the scientific validation of urine. It is important to document this sufficiently upfront to ensure correct sampling conditions can be used (including potentially addition of solubilizing agents) prior to starting the clinical study.

\section{Tissue homogenates}

Tissue (homogenate) analysis, categorized as assay appropriate scientific validation, involves multiple considerations from a bioanalytical perspective. Often, tissues are analyzed as part of understanding relative exposure ratios and answering these questions may be subject to more simple screening approaches. A comprehensive EBF recommendation for the analysis 
Table 2. Proposed criteria for in-study acceptance for the analysis of metabolites (in relation to ICH M3 [R2]), urine and tissue analysis.

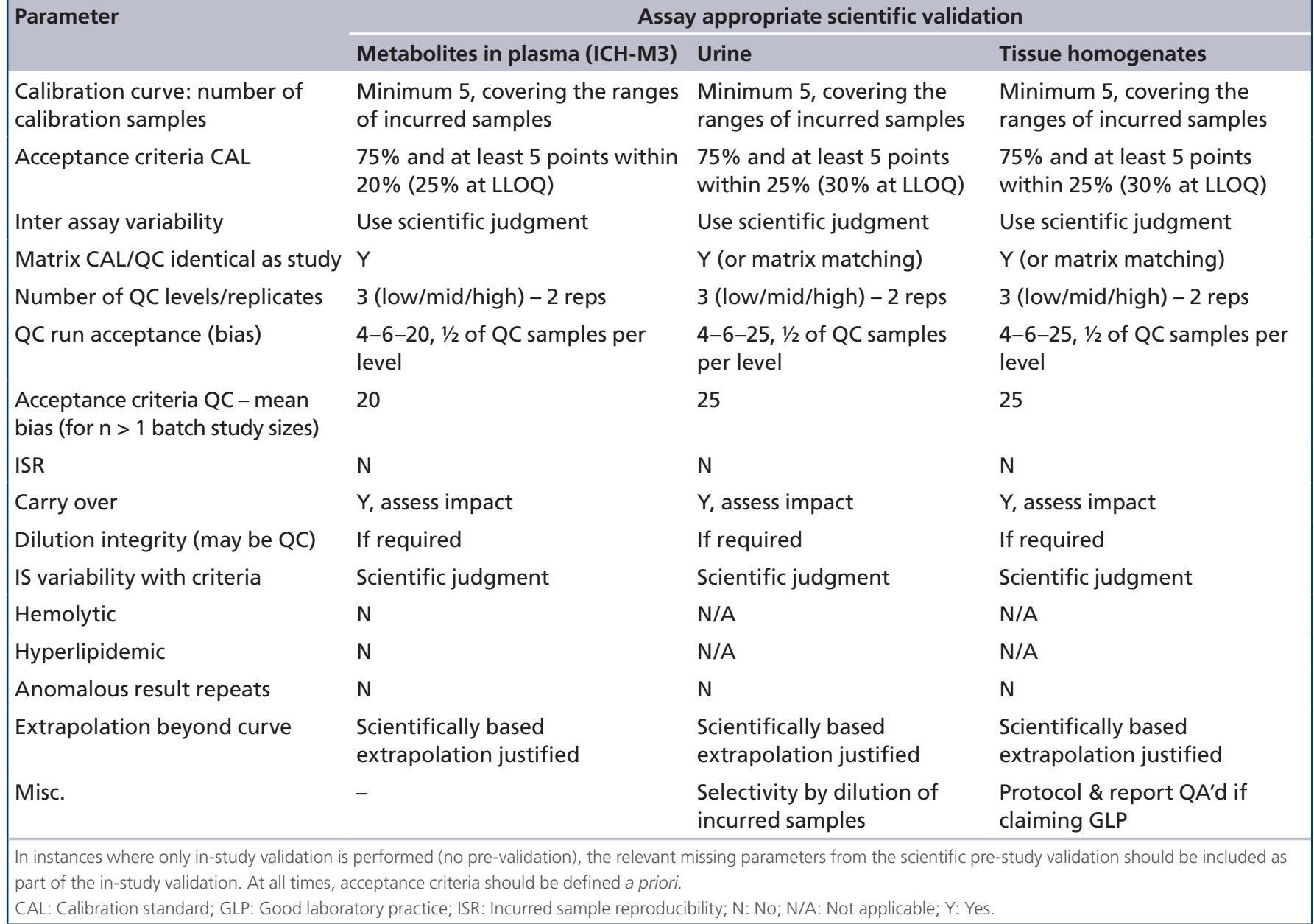

of tissue homogenates was published earlier [20]. However, as mentioned in the introduction, no detailed guidance was provided on what scientific validation criteria look like in case absolute tissue (homogenate) concentrations are requested. Prior to embarking on the actual analysis, we recommend having the discussion with the requester of the concentration data if absolute concentrations are really needed. In the latter case, EBF recommends using to use the scientific validation criteria proposed in this manuscript.

\section{Documentation}

Documentation is an essential part of best scientific validation practices. Reconstructability of results and decisions taken throughout the assay development, assay validation or during production should be common practice and it is our recommendation to apply best documentation practice. At the same time, it is important that decisions are not made by coincidence, but can be made based on sound scientific criteria which are a priori defined in a protocol or a standard operating procedure and later reported in the raw data or a study report.

\section{Standard operating procedures}

As a result of our discussions and in line with regulatory requirement referring to the requirements for bioanalytical testing [25-29], we would recommend that the bioanalytical laboratory develops (a) dedicated SOP(s) to describe and manage the scientific validation practices. This SOP should describe the principles of tiered approach, define the scope or the studies/assays for which the SOP can be applied and provide experimental details on the practical execution of scientific validation for each of the assay types in scope. This manuscript is intended to be the basis of this SOP, in a way that it allows the industry to come together on the practical and harmonized execution of scientific method validation for urine, tissues, metabolite (related to ICH M3[R2]), nonpivotal clinical or preclinical studies. If deemed more appropriate, the SOP(s) can be replaced by a bioanalytical study protocol providing similar guidance. 
Reporting \& archiving

With respect to reporting and archiving, we recommend being mindful of the resources required to report the concentration data, and work towards a process which combines transparent reporting of these data and the performance of the assay which was used

Table 3. Proposed criteria for pre-study method validation for the analysis of early development preclinical studies or clinical studies.

\begin{tabular}{|c|c|c|}
\hline \multirow[t]{2}{*}{ Parameter } & \multicolumn{2}{|c|}{ Stage appropriate scientific validation } \\
\hline & Early development clinical studies & Early development preclinical studies \\
\hline $\begin{array}{l}\text { CoA with at minimum proof of } \\
\text { identity/purity }\end{array}$ & Y or use dosed batch & Y or use dosed batch \\
\hline $\begin{array}{l}\text { Calibration curve: number of } \\
\text { calibration samples }\end{array}$ & $\begin{array}{l}\text { Minimum 6, covering the ranges of } \\
\text { incurred samples }\end{array}$ & $\begin{array}{l}\text { Minimum 6, covering the ranges of } \\
\text { incurred samples }\end{array}$ \\
\hline Acceptance criteria CAL & $\begin{array}{l}75 \% \text { and at least } 6 \text { points within } 20 \% \\
(25 \% \text { at LLOQ) }\end{array}$ & $\begin{array}{l}75 \% \text { and at least } 6 \text { points within } 20 \% \\
(25 \% \text { at LLOQ) }\end{array}$ \\
\hline Matrix CAL/QC identical as study & $\mathrm{Y}$ & Y \\
\hline QC levels - replicates & 4 (LLOQ/low/mid/high) - min. 5 reps & 4 (LLOQ/low/mid/high) - $\min .5$ reps \\
\hline Acceptance criteria QC - mean bias & $20 \%(25 \%$ at LLOQ $)$ & $20 \%(25 \%$ at LLOQ $)$ \\
\hline Acceptance criteria QC (\%CV per level) & $20 \%(25 \%$ at LLOQ $)$ & $20 \%(25 \%$ at LLOQ $)$ \\
\hline Inter assay variability & $\begin{array}{l}\text { Use scientific judgment based upon P\&A of } \\
\text { 1-run validation }\end{array}$ & $\begin{array}{l}\text { Use scientific judgment based upon P\&A } \\
\text { of 1-run validation }\end{array}$ \\
\hline QC/CAL from separate stocks & Y, unless accuracy of stock proven & Y, unless accuracy of stock proven \\
\hline Selectivity & 6 & $\mathrm{n}=1$ matrix source is still relevant \\
\hline Extraction recovery & $\mathrm{N}$ & $\mathrm{N}$ \\
\hline Carryover & In study & In study \\
\hline Matrix effect & $\mathrm{N}$, assess within study runs via IS response & $\mathrm{N}$, assess within study runs via IS response \\
\hline Dilution integrity & In study & In study \\
\hline LLOQ & $\begin{array}{l}\text { As defined by acceptable LLOQ QC } \\
\text { standard }\end{array}$ & $\begin{array}{l}\text { As defined by acceptable LLOQ QC } \\
\text { standard }\end{array}$ \\
\hline $\begin{array}{l}\text { Comed selectivity (in support of DDI } \\
\text { studies) }\end{array}$ & $\mathrm{N}$ & $\mathrm{N}$ \\
\hline Over the counter stability & $\mathrm{N}$ & $\mathrm{N}$ \\
\hline Fixed dose combination stability & $\mathrm{N}$ & $\mathrm{N}$ \\
\hline $\begin{array}{l}\text { Processed sample stability/ } \\
\text { reproducibility }\end{array}$ & Scientific judgment & Scientific judgment \\
\hline Stock solution stability & Y, unless prepared the same day. & Y, unless prepared the same day. \\
\hline $\begin{array}{l}\text { Bench-top stability; sample stability } \\
\text { for duration of storage; F/T stability }\end{array}$ & $\begin{array}{l}\text { Consider combined stability experiment to } \\
\text { cover unknown samples }\end{array}$ & $\begin{array}{l}\text { Consider combined stability experiment to } \\
\text { cover unknown samples }\end{array}$ \\
\hline Whole blood stability & N, unless for known problem scaffolds [22] & N, unless for known problem scaffolds [22] \\
\hline Sampling conditions & Cover via a combined stability expt & Cover via a combined stability expt \\
\hline Hemolytic & $\mathrm{N}$ & $\mathrm{N}$ \\
\hline Hyperlipidemic & $\mathrm{N}$ & $\mathrm{N}$ \\
\hline Validation plan/protocol & $\begin{array}{l}\text { At minimum SOP or short protocol } \\
\text { summarizing predefined scientific of } \\
\text { parameters tested }\end{array}$ & $\begin{array}{l}\text { At minimum SOP or short protocol } \\
\text { summarizing predefined scientific of } \\
\text { parameters tested }\end{array}$ \\
\hline Validation report & $\begin{array}{l}\text { At minimum a document summarizing } \\
\text { scientific parameters tested }\end{array}$ & $\begin{array}{l}\text { At minimum a document summarizing } \\
\text { scientific parameters tested }\end{array}$ \\
\hline
\end{tabular}


Table 4. Proposed criteria for in-study acceptance for the analysis of early development preclinical studies or clinical studies.

Parameter

Stage appropriate scientific validation

Early development clinical studies

Minimum 6, covering the ranges of incurred samples

Calibration curve: number of calibration samples

Acceptance criteria CAL

$75 \%$ and at least 6 points within $20 \%$ (25\% at LLOQ)

Inter-assay variability Use scientific judgment

Matrix CAL/QC identical as study $Y$

Number of QC levels/replicates 3 (low $/ \mathrm{mid} / \mathrm{high}$ ) - 2 reps

4-6-20, $1 / 2$ of QC samples per level

QC run acceptance (bias)

$20 \%$

Acceptance criteria QC - mean
bias (for $n>1$ batch study sizes)

\begin{tabular}{|c|c|c|}
\hline ISR & Y, once & Y, once per species \\
\hline Carry over & Y, assess impact & Y, assess impact \\
\hline Dilution integrity (may be QC) & If required & If required \\
\hline IS variability with criteria & Refer to [23] & Refer to [23] \\
\hline Hemolytic & $\begin{array}{l}\mathrm{N} \text {, is part of IS tracking, observation recorded } \\
\text { by clinical unit - interrogate anomalous results }\end{array}$ & $\begin{array}{l}\mathrm{N} \text {, is part of IS tracking - interrogate } \\
\text { anomalous results }\end{array}$ \\
\hline Hyperlipidemic & $\mathrm{N}$, is part of IS tracking & N/A \\
\hline Anomalous result repeats & Yes, as requested in duplicate & Yes, as requested in duplicate \\
\hline Extrapolation beyond curve & Scientifically based extrapolation justified & Scientifically based extrapolation justified \\
\hline Repeat if drug in placebo/control & Yes in duplicate & Yes in duplicate \\
\hline
\end{tabular}

to generate them. Also, as an industry we should be able to cope with the emotion that, for the sake of argument, a study costing 25,000 can be summarized on three pages. So, keeping it brief and relevant is our proposal. As part of our discussion in preparation of the current recommendation paper, we reflected on proposing a report template, but limit our proposal to the minimum required information listed below. We would expect a study or validation report to contain:

- Introduction:

- Reference to study number, protocol or SOP (may include signature of sponsor);

- Introduction explaining the scope of scientific validation to frame the context.

- Body:

- Short assay description or reference to the assay description;
Early development preclinical studies

Minimum 6, covering the ranges of

incurred samples

$75 \%$ and at least 6 points within $20 \%$

(25\% at LLOQ)

Use scientific judgment

Y

3 (low/mid/high) - 2 reps

$4-6-20,1 / 2$ of QC samples per level

$20 \%$

once per species

, assess impact

Refer to [23]

$\mathrm{N}$, is part of IS tracking - interrogate

results

Yes, as requested in duplicate

Scientifically based extrapolation justified

- Short summary table providing evidence of assay performance, range and stability;

= More detail can be added in an appendix as required or as per company desire (e.g., sponsor-vendor relationship).

- There would be no need to include chromatograms;

- No GLP claim on validation;

- A GLP claim should remain possible on the study data if so required;

- Signature of study responsible person.

Although we suggest to limit reporting of tissue homogenate analysis to a document summarizing scientific parameters test, in case of tissue homogenate analysis on tissues originating from a GLP study claiming GLP, a report containing the parameter proposed in this paragraph is recommended. All raw data required to reconstruct the study data 
should be archived. Duration of archiving should be aligned with agreed record retention schedules, company policies, contracts between vendor-sponsor and in accordance with regulations.

\section{Conclusion}

Tiered approach is an important development in regulated bioanalysis. As was the case in the early nineties, when the industry came together to propose harmonized criteria for the main bioanalytical studies being analyzed in that era, it is important we join hands again to agree on applying the principles of tiered approach in a harmonized way. Good science and sustainable use of resource are key. Discussion in industry has shown that differentiating tiered approach into different levels of quality is a desired practice. By proposing detailed and practical criteria for a major part of early development studies, which have been discussed with and agreed by many scientist, not only in the EBF, it is our firm belief that starting to use these criteria in a harmonized way will give the necessary scientific freedom and optimized use of resource to generate data with the appropriate scientific validity to make valid decisions. At the same time, by using these alternative approaches in the proposed earlier stages of development, it can contribute to an easier adoption of the regulatory guidance requirements for the studies were they were intended for. Indeed, applying these criteria and performing all the required experiments will be less challenging if this can be limited to the portfolio of compounds still under development in these later stages of development versus the longer list of compounds that will never reach the patient.

\section{Future perspective}

Although the principles of tiered approach have been around for more than a decade, implementation in the bioanalytical lab is becoming increasingly important.

\section{References}

1 Viswanathan CT et al. Workshop/Conference Report - Quantitative bioanalytical methods validation and implementation: best practices for chromatographic and ligand binding assays. AAPS J. 9(1), 1962-1973 (2007).

2 Timmerman P, Kall M, Gordon B et al. Best practices in a tiered approach to metabolite quantification: views and recommendations of the European Bioanalysis Forum. Bioanalysis 2(7), 1185-1194 (2010).

3 Lowes S, Hucker R, Jemal M et al. Tiered approaches to chromatographic bioanalytical method performance evaluation: recommendation for best practices and harmonization from the Global Bioanalysis Consortium Harmonization Team. AAPS J. 17(1), 17-23 (2015).
The reasons are at least twofold: greater focus on science and smarter use of resources. This combination potentially leading to better and safer drugs brought faster and more cost effectively to the patient. In addition, it is our strong belief that by embracing the principles of scientific validation as a complementary approach to regulatory validation, especially for a variety of early development studies where scientific validation allows better focus on the scientific questions asked, will ensure a more harmonized interpretation and application of different bioanalytical Guidance or Guidelines in later stages of development. For the near future, EBF is committed to take appropriate initiatives to continue the discussion and to develop similar strategic thinking for other areas of bioanalytical support beyond the 5 focus areas in the current recommendation paper.

\section{Disclaimer}

The views and conclusion presented in this article are those of the European Bioanalysis Forum's topic team and do not necessarily reflect the representative affiliation or company's position on the subject.

\section{Acknowledgements}

The authors acknowledge the European Bioanalysis Forum (EBF) member companies that have contributed to the surveys, workshops and internal discussions, which form the backbone of the current recommendation.

\section{Financial \& competing interests disclosure}

The authors have no relevant affiliations or financial involvement with any organization or entity with a financial interest in or financial conflict with the subject matter or materials discussed in the manuscript. This includes employment, consultancies, honoraria, stock ownership or options, expert testimony, grants or patents received or pending, or royalties.

No writing assistance was utilized in the production of this manuscript.

4 Booth B. When do you need a validated assay? Bioanalysis 3(24), 2729-2730 (2011).

5 Aubry AF, Christopher L, Wang J et al. Reflecting on a decade of metabolite screening and monitoring. Bioanalysis 6(5), 651-664 (2014).

6 Abbott R. Tiered approach: sense and sensibility. Bioanalysis 6(5), 611-616 (2014).

7 Guidance for Industry Bioanalytical Method Validation U.S. Department of Health and Human Services Food and Drug Administration Center for Drug Evaluation and Research (CDER) Center for Veterinary Medicine (CVM) (2001). www.ema.europa.eu

8 EMEA/CHMP/EWP/192217/2009, Guideline on bioanalytical method validation. www.ema.europa.eu 
9 MHLW Guideline (Japan). http://bioanalysisforum.jp

10 CPMP/ICH/286/95 - ICH Topic M 3 (R2) Non-Clinical Safety Studies for the Conduct of Human Clinical Trials and Marketing Authorization for Pharmaceuticals. www.ema.europa.eu

11 Viswanathan C. Bringing new technologies into regulatory space. Bioanalysis 4(23), 2763-2764 (2012).

12 Timmerman P, Henderson N, Smeraglia J et al. Managing scientific, technical and regulatory innovation in regulated bioanalysis: a discussion paper from the European Bioanalysis Forum. Bioanalysis 5(2), 139-145 (2013).

13 FDA Draft Guidance for Industry Bioanalytical Method Validation. www.fda.gov

14 Mini focus 1 issue Bioanalysis on tiered approach. www.future-science.com

15 Timmerman P. Tiered approach revisited: introducing stage-appropriate or assay-appropriate scientific validation. Bioanalysis 6(5), 599-604 (2014).

16 Timmerman P, White S, Knutsson M et al. Feedback from the European Bioanalysis Forum Workshop: taking tiered approach to the next level. Bioanalysis 6(19), 2593-2598 (2014).

17 Taking tiered approach to the next level feedback from the EBF workshop. http://bcn2014.europeanbioanalysisforum.eu

18 Timmerman P, Lowes S, McDougall S et al. Scientific or regulated validation - a tiered approach? Meeting report from a Joint EBF/DVDMDG Workshop, Bioanalysis 7 (14), 1703-1710 (2015).

19 Xue Y-J, Gao H, Ji QC et al. Bioanalysis of drug in tissue: current status and challenges. Bioanalysis 4(21), 2637-2653 (2012).
20 Timmerman P, Mokrzycki N, Delrat P et al. Recommendations from the European Bioanalysis Forum on method establishment for tissue homogenates. Bioanalysis 6(12), 1647-1656 (2014).

21 Clinical Leader. Pharmaceutical Benchmarking Forum's Success Rate Data Illuminates Challenges to R\&D Industry. www.clinicalleader.com

22 Freisleben A, Brudny-Klöppel M, Mulder $\mathrm{H}$ et al. Blood stability testing: European Bioanalysis Forum view on current challenges for regulated bioanalysis. Bioanalysis 3(12), 1333-1336 (2011).

23 White $\mathrm{S}$, Adcock N, Elbast W et al. European Bioanalysis Forum: recommendation for dealing with internal standard variability. Bioanalysis 6(20), 2767-2774 (2014).

24 Rocci ML Jr, Collins E, Wagner-Caruso KE et al. Investigation and resolution of incurred sample reanalysis failures: two case studies. Bioanalysis 3(9), 993-1000 (2011).

25 FDA Department of Health and Human services, CFR - Code of Federal Regulations Title 21 part 58, Good Laboratory Practice Regulations, Final Rule (1987). www.gpo.gov

26 OECD GLP. www.oecd.org

27 FDA Department of Health and Human services, CFR - Code of Federal Regulations Title 21 part 320.29 Analytical methods for an in vivo bioavailability or bioequivalence.

www.gpo.gov

28 ICH S3A.

www.ich.org

29 ICH E6.

www.fda.gov 